KALMAN FILTER BASED FAULT DETECTION AND REPLACEMENT SIGNAL GENERATION OF SENSOR MEASUREMENTS IN CEMENT ROTARY KILN

\author{
V. GOMATHI ${ }^{1}$, K. RAMKUMAR ${ }^{2}$, GURUPRASATHMURALIDHARAN ${ }^{3}$ \\ \& SESHADHRISRINIVASAN ${ }^{4}$ \\ ${ }^{1,2}$ SASTRA Deemed University, Thanjavur, Tamilnadu, India \\ ${ }^{3}$ Smarta Opti Solutions, Chennai, Tamilnadu, India
}

${ }^{4}$ Berkeley Education Alliance for Research in Singapore, Singapore

\begin{abstract}
Replacement of faulty sensor signals in a cement kiln is a challenging problem in fault diagnosis due to the increasing demand of fault- free feedback signal to the controller in order to produce good quality cement and estimation technique have been widely practiced for this. It is found from the literature that sensor faults in a highly energyintensive process will have a huge impact on the end product quality and profits. Cement industries, particularly the rotary kiln processes are plagued by temperature sensor faults, oxygen analyzer faults and it is a significant engineering research problem to diagnose the fault accurately amidst both random sensor and system disturbances. Fault in these measurements will severely affect the controller performance resulting in poor quality cement. In this work, Kalman filter based residual signal generation and replacement signal generation technique is employed for detecting bias fault and sensor measurement failure in pyrometer, thermocouple, and preheater oxygen analyzer of the kiln process because of the significance of these measurements in determining the quality of the cement. This work is divided into threephase1) Modelling 2) Residual signal generation 3) Replacement signal generation. The subspace method based state space model has been identified for three sensors and the residuals are found by finding the difference between the estimated states from the Kalman filter and the actual states and the deviation in the residuals will be used as a metric to indicate the nature and occurrence of faults, finally replacement signal has been generated for faulty sensors using the prior estimated states with irrespective of the sensor measurement.
\end{abstract}

KEY WORDS: Rotary Kiln, Kalman Filter, Residual Generation \& Replacement Signal

Received: Jan 31, 2018; Accepted: Feb 21, 2018; Published: Mar 13, 2018; Paper Id: IJMPERDAPR201885

\title{
INTRODUCTION
}

Industrial statistics show that about $70 \%$ of the industrial accidents are caused by faults or failure in the system components and the chemical industries lose approximately 20 billion dollars every year due to the failure of components. The component failure will lead faulty feedback signal which may cause poor controller performance, so abnormal event management is the major problem in all the industries and the process supervision is an indispensable technical necessity to take key decisions to avoid hazardous and unacceptable events related to failure or damage to the equipment, operator safety or any accidents. Generally, faults are identified as the deviation of process variables from the actual and this deviation once supervised and tracked can lead to better control and optimization decisions. Normally in an industry, this supervision is done by fault detection and 
isolation (FDI) system. In the past few decades, many FDI system has been developed for process monitoring and it is widely reported and discussed in the literature [6]. Many of the industrial processes can be modeled to be a good level of accuracy and so model -based fault diagnosis techniques are quite popular and these methods are classified into the quantitative model based method, qualitative model -based method and process history based method. (i) Qualitative models have been developed based on the knowledge about the physics of input/output relations of the process, it develops as causal models, abstraction hierarchy [16] (ii) Quantitative methods like observer, parity equations, Kalman filter discussed in detail [18] and these methods need complete mathematical relationship between input and output.(iii) Process history based techniques like the neural network, principal component analysis, partial least square etc were also elaborately discussed [17]. Based on the location of occurrence, the fault is classified into actuator fault, sensor fault ,and process fault. The sensor fault detection is the major area reported in detail in the literature. The sensor fault detection in real time applications has been analyzed elaborately in many research works. Faults in magnetometer used in satellites were detected by robust extended Kalman filter, robust unscented Kalman filter using multi scale adaptation technique but it needs more computational load [15] and the Kalman gain have also been computed by proportional integral action for stochastic and deterministic system by convex optimization methods [8], moreover this algorithm has also been used for sensor fault, actuator fault detection in CSTR process for achieving high robustness against model uncertainty[4] and for de-signing observer gain for piezoelectric actuated flexible beam, which give high robustness towards uncertainty of hysteresis [12]. The ensemble Kalman algorithm were used for detecting sensor fault using multi -sensor decentralized fusion and multi- sensor decentralized synchronous and asynchronous methodology [21]. Fault detection in nonlinear system has also been studied using EKF and it gives better results than linear Kalman filter, but it needs more computational time [10]and this technique have been used for the analysis of buffer tank system for detecting sticky, stuck and drift faults by considering time of occurrence as one parameter for finding fault model, but this increases the computational burden [19]. The convergence time for detecting sensor faults have been reduced in spacecraft application by the fast converging EKF with suitable initialization of covariance matrix [7]. With these Kalman filter based fault detection techniques for generating the residuals, fault tolerant controller was also designed for linear drives [5].The reliability of sensor has also been improved in engine control system by detecting hard failures like out of range and large bias using self -tuning Kalman filter and it does not need accurate model and statistical properties of noise [20] and this algorithm combined with pattern recognition to predict the future states for finding faults by suitable feature selection in current and voltage sensors of induction motor [11], but number of features will increase the computation time and decrease the accuracy. In flight control system, sensor fault and actuator fault are determined by testing generalized Rayleigh quotient for innovation covariance of Kalman filter [3], sensor faults have also been detected in CSTR process using soft sensor, but in this technique variance of unmeasured states is not equal to variance of actual process variable [13]. Extreme learning algorithm has been tried for detecting faults and generating replacement signal in burning zone temperature sensor used in kiln process to improve the quality of cement and to increase the productivity [9], Extended Kalman filter based sensor fault detection and isolation and Luenberger observer based recovery signal generation have also been discussed in [1] for interior permanent magnet synchronous motor with sensorless drive and it is inferred that this technique has not been used for very low -speed applications and for multiple sensor faults. Kalman filter used for generating replacement signal with intermittent observations is discussed in [14] and the binomial variable is chosen as dummy measurement during sensor fault or failure.

In this work Kalman filter based fault detection method is proposed to detect measurement faults and to generate the replacement signal for faulty sensors without the inclusion of any new binomial variable in the correction stage for 
burning zone temperature sensor (Pyrometer), kiln inlet temperature sensor (Thermocouple) and preheater oxygen analyzer (Oxygen analyzer) and this signal have been generated by considering the prior estimated states and neglecting posterior estimated states under constant input.

The chapters are organized as follows: chapter II gives the description about the process and the mathematical model of the kiln sensors are described in chapter III, Kalman algorithm for fault detection and replacement signal generation are explained in chapter IV, chapter V presents the simulation results and this work is concluded in chapter VI.

\section{PYRO PROCESS}

Cement is a substance, produced by three important stages, preparation of raw materials, the formation of clinkers (pyro process) and grinding. In these three stages, pyro processing plays a major role, because rotary kiln used in this pyro process is considered as the heart of the cement plant. The kiln is used for burning the precalcined material and it leads many chemical reactions and finally, clinkers formed with a grey color spherical ball with $0.32 \mathrm{~cm}-0.5 \mathrm{~cm}$ diameter. There are three important stages on pyro- processing. (1) Preheating (2) Calcining (3) Burning. The preheater consists of many cyclones, through which the raw material is given. The raw materials are mixed in the following proportionCaCO $\mathrm{CO}_{3}(80 \%)$, $\mathrm{SiO}_{2}(13 \%), \mathrm{Al}_{2} \mathrm{O}_{3}(3 \%), \mathrm{Fe}_{2} \mathrm{O}_{3}(2 \%), \mathrm{MgO}(1.6 \%)$. Hot gas from the combustion process comes and contact with the raw material and evaporating water from the mixture. At each stage of the preheater, the mixture is heated to a particular temperature, at last stage $70 \%$ of precalcination will be completed and temperature is maintained around $900^{\circ} \mathrm{C}$ and this feed is given to calciner where the complete decarbonation takes place.Equation 1 shows the conversion of lime stone into lime (decarbonation).

$$
\mathrm{CaCO}_{3} \rightarrow \mathrm{CaO}+\mathrm{CO}_{2}
$$

Calcined feed (around $1000^{\circ} \mathrm{C}$ ) is given to the higher end of the rotary kiln and fuel is given to lower end for burning the feed to very high temperature. Since the kiln is inclined, the material will be moved from higher end to lower end, at this end the temperature is around $1450^{\circ} \mathrm{C}$, later this material is moved to grate cooler, where the clinker is formed with the temperature of around $100^{\circ} \mathrm{C}$.Finally, the clinker is milled and mixed with gypsum to produce cement [2] and it shown has in Equation 2

$$
\mathrm{Ca}_{2} \mathrm{SiO}_{4}+\mathrm{CaO} \rightarrow \mathrm{Ca}_{3} \mathrm{SiO}_{5}
$$

The process flow of kiln is shown in Figure1. If the reaction is incomplete, an excessive amount of $\mathrm{CaO}$ remains in the clinker, various gases are emitted in the above process, $\mathrm{CO}_{2}$ is emitted as a result of decarbonation, $\mathrm{NOx}$ is emitted as it is a hightemperature process, $\mathrm{O}_{2}$ is emitted if there is excess oxygen given for combustion. Moreover, burning zone temperature and kiln inlet temperature are also very important parameters to be monitored because of clinker quality changes based on that.So, monitoring of gas analyzers and temperature sensors is very important, a fault in these sensors will affect the controller performance such a way that fluctuates the temperature inside the kiln, surrounding environment and systems may affect, poor quality clinker produces, energy dissipates, ring forms around the surface of the kiln which reduces the diameter of the kiln. Thus, an overall efficiency of the plant reduces due to a single fault in a system, so it needs to be identified and replacement signal should be generated immediately in order to avoid the poor controller performance. 


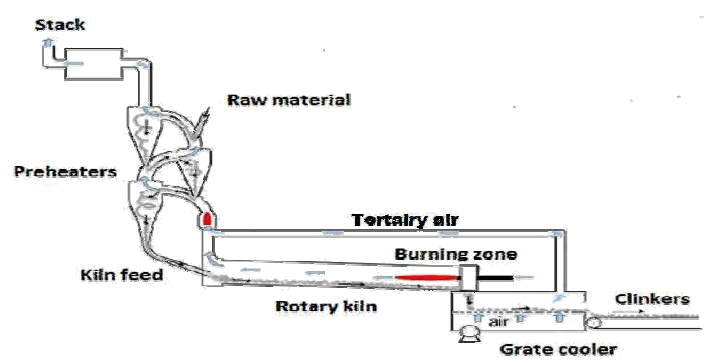

Figure 1: Flow Diagram of Pyroprocess

\section{MATHEMATICAL MODELLING}

Since Kalman algorithm needs the mathematical model for analysis, it is necessary to find the model of the kiln sensors. Initially, the data were collected for kiln feed and preheater inlet oxygen $\left(\mathrm{O}_{2}\right)$, Burning zone temperature (BZT),Kiln inlet temperature (KIT) from cement process station. Subspace method based state space model for kiln sensors have been identified using system identification tool in MATLAB. The state space model of the kiln sensors is given below

$$
\begin{aligned}
A & =\left[\begin{array}{cccc}
0.9994 & 0.0047 & 0.0035 & 0.0012 \\
-0.0015 & 0.9937 & 0.0518 & -0.0063 \\
0.0208 & -0.1044 & 0.7425 & 0.0579 \\
0.0110 & -0.0050 & -0.3489 & 0.9172
\end{array}\right] \\
B & =\left[\begin{array}{c}
-0.0006 \\
-0.0051 \\
0.0265 \\
0.0447
\end{array}\right] \\
C & =\left[\begin{array}{cccc}
88.7579 & 10.7692 & -0.1066 & 0.0683 \\
160.5605 & -13.8046 & 0.0476 & -0.0050 \\
1.6009 & 0.0542 & -0.3229 & 0.0146
\end{array}\right] \\
D & =\left[\begin{array}{l}
0 \\
0 \\
0
\end{array}\right], X 0=\left[\begin{array}{ccc}
10.249 \\
9.7823 \\
44.501 \\
28.906
\end{array}\right]
\end{aligned}
$$

\section{KALMAN FILTER ALGORITHM}

Now a days the role of state estimators for real time fault diagnosis system has been increased. In this work, Kalman filter based state estimator is used for identifying the faults and generating replacement signal for the faulty sensor measurements in order to improve the controller performance during the faulty conditions. Since this estimator needs system model, datadriven based subspace method is used for identifying the state space model of the sensors used in the kiln. The discrete time LTI system is governed by Equation 3 and 4

$$
\begin{aligned}
& x(k+1)=A x(k)+B u(k)+Q w(k) \\
& y(k)=C x(k)+R v(k)
\end{aligned}
$$

Where $A$ is system matrix, $B$ is input matrix, $C$ is output matrix, $x(k)$ is state vector, $u(k)$ is control vector, $y(k)$ is measurement vector. The process noise and measurement noise $w(k), v(k)$ shown in the equation are uncorrelated 
Gaussian random noise with zero mean and $Q, R$ covariances. There are two stages in the Kalman algorithm (i.e) prediction and correction. In the prediction stage, the prior states are estimated shown in Equation 5 and compared with the actual states for finding the estimation error, which is necessary to find the prior error covariance shown in Equation 6

$$
\begin{aligned}
& x(k+k \mid k)=A x(k \mid k)+B u(k \mid k) \\
& p(k+1 \mid k)=A p(k \mid k) A^{T}+Q
\end{aligned}
$$

wherep $(k)=E\left[e(k) e(k)^{T}\right] ; e(k)=x(k)-\hat{x}(k)$ The prior states and error covariance are used in the correction stage for updating Kalman gain, posterior state and posterior error covariance shown in Equations 7,8 and 9

$$
\begin{aligned}
& K(k+1)=p(k+1 \mid k) C^{T}\left[C p(k+1 \mid k) C^{T}+R\right]^{-1} \\
& \hat{x}(k+1 \mid k+1)=\hat{x}(k+1 \mid k)+K(k+1[y(k+1-\hat{y}(k+1 \mid k)] \\
& p(k+1 \mid k+1)=[I-K(k+1) C] p(k+1 \mid k)[I-K(k+1) C]^{T} \\
& +K(k+1) R K(k+1)^{T}
\end{aligned}
$$

Kalman filter has been used to estimate all 3 sensor states by properly tuning process noise covariance $(Q)$ and measurement noise covariance $(R)$. In this algorithm $Q, R$ was tuned based on the observation of minimum residual signal and this residual is computed as the difference between actual measurements and estimated outputs. The residual will be used as the key for detecting faults and it is zero under faultfree condition and deviate from zero when fault or disturbance occurs. If the residual signal is less than the threshold but deviates from zero then it is concluded that the deviation is due to disturbance. The fault can be concluded when the residual signal is greater than the threshold value. From the proposed method it is observed that the estimation follows the prediction, not the correction (i.e) instead of believing measurements it is believing model output during faulty condition. Finally, the predicted output will be used as the replacement signal. Figure 2 shows the block diagram of Kalman filter based FDI system. Where $\mathrm{U}$ is kiln feed rate and $Y$ is $Y_{1}, Y_{2}, Y_{3}$ which are preheater inlet oxygen $\left(\mathrm{O}_{2}\right)$, Burning zone temperature(BZT), Kiln inlet temperature(KIT).

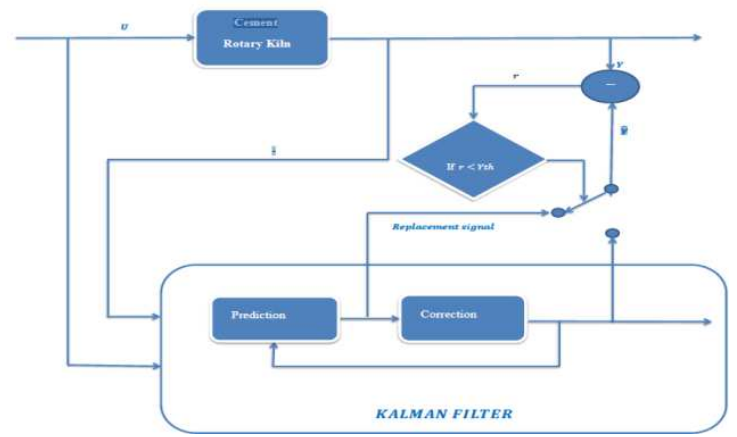

Figure 2: Block Diagram of Kalman Filter Based FDI System

\section{SIMULATION RESULTS}

Initially, the data were collected for 7 hours duration with 10 seconds sampling interval from the cement rotary kiln using cement process station and these data were used to identify the state space model of sensors using system identification tool in MATLAB .Finally, this model have been used in Kalman algorithm for detecting sensor faults and to generate replacement signal. Using this algorithm all 3 sensor states has been predicted, estimated and the estimated 
output was compared with the actual measurements to find the residual signals in order to detect the occurrence of fault in sensors and to generate replacement signal from the predicted output and by neglecting the faulty sensor measurements.

The residual signal for the sensor measurements under the fault free condition is given in Equation 10, 11 and 12

$$
\begin{aligned}
& r_{O_{2}}=Y_{1}-\widehat{Y}_{1}^{+} \\
& r_{B Z T}=Y_{2}-\widehat{Y}_{2}^{+} \\
& r_{K I T}=Y_{3}-\widehat{Y}_{3}^{+}
\end{aligned}
$$

The residual signal for the sensor measurements under faulty condition is given in Equation 13, 14 and 15

$r_{O_{2}}=Y_{1}-\widehat{Y}_{1}^{-}$

$r_{B Z T}=Y_{2}-\widehat{Y}_{2}^{-}$

$r_{K I T}=Y_{3}-\widehat{Y}_{3}^{-}$

$\mathbf{r}_{\mathbf{O}_{2}}:$ Residual signal of preheater inlet oxygen

$\mathbf{r}_{\mathrm{BZT}}$ : Residual signal of burning zone temperature

$\mathbf{r}_{\mathrm{KIT}}$ : Residual signal of kiln inlet temperature

$\mathbf{Y}_{1}$ : Preheater inlet oxygen

$\mathbf{Y}_{2}$ : Burning zone temperature

$\mathbf{Y}_{3}$ : Kiln inlet temperature

$\widehat{\mathrm{Y}}_{1}^{+}$: Posteriori preheater inlet oxygen

${\widehat{Y_{2}}}^{+}$: Posteriori burning zone temperature

${\widehat{\mathbf{Y}_{3}}}^{+}$: Posteriori kiln inlet temperature

${\widehat{\mathrm{Y}_{1}}}^{-}$: Priori preheater inlet oxygen

${\widehat{\mathrm{Y}_{2}}}^{-}$: Priori burning zone temperature

${\widehat{\mathrm{Y}_{3}}}^{-}$: Priori kiln inlet temperature

Threshold level for detecting fault in sensor is given in Equation 16, 17 and 18

$$
\begin{aligned}
& Y_{1 t h}=Y_{1 \text { nominal }} \pm 5 \\
& Y_{2 t h}=Y_{\text {2nominal }} \pm 100 \\
& Y_{3 \text { th }}=Y_{3 \text { nominal }} \pm 100
\end{aligned}
$$

$\mathbf{Y}_{\mathbf{1 t h}}$ : Threshold level of Preheater inlet oxygen

$\mathbf{Y}_{\mathbf{2 t h}}$ : Threshold level of burning zone temperature 
$\mathbf{Y}_{3 \text { th }}$ : Threshold level of kiln inlet temperature

Figure3 shows three sensors estimated output, measurement variations and model output for kiln feed change given at the input and it shows that the estimated signal follows the model output and measurement signal changes exactly under fault free condition. Its residual signal is shown in Figure 4 and it is zero, so it is concluded that there is no fault occurs in any of the sensors.
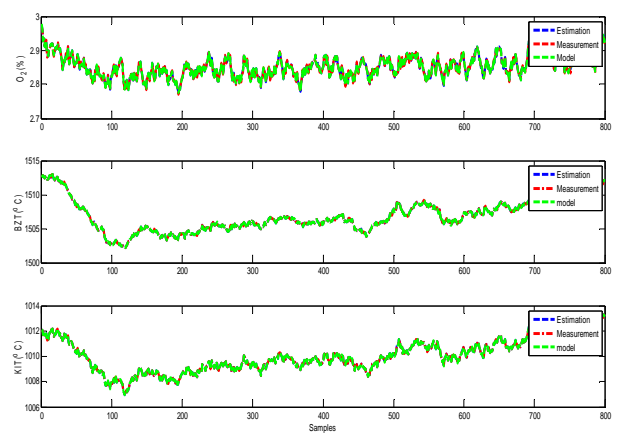

Figure 3: Sensor Measurements under Fault Free Condition
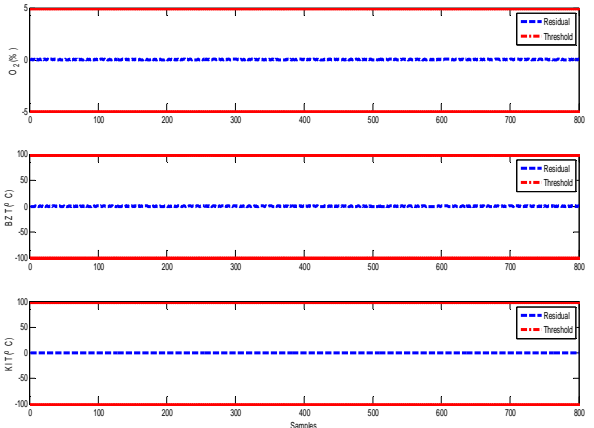

Figure 4: Residual Signals under Fault Free Condition
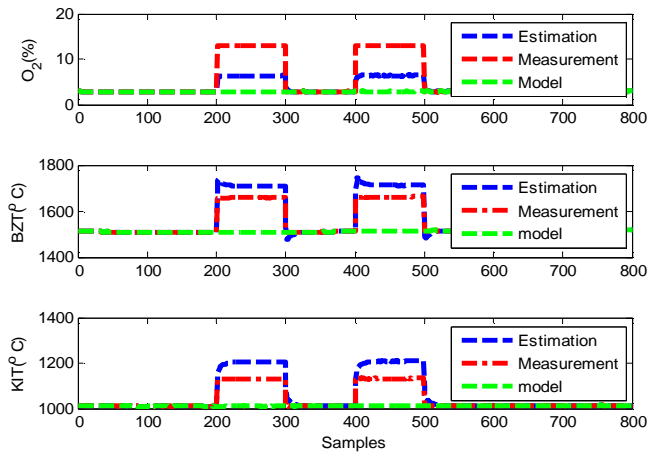

Figure 5: Sensor Measurements under Faulty Condition Using Conventional Kalman Algorithm 

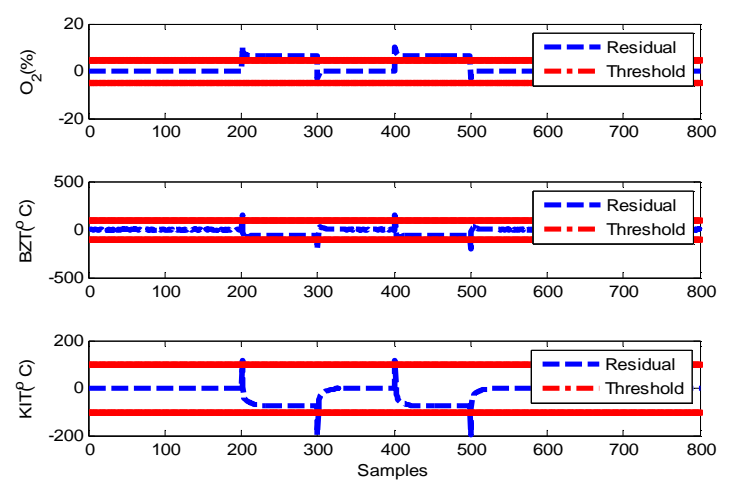

\section{Figure 6: Residual Signals under Faulty Condition Using Conventional Kalman Algorithm}

Figure 5 shows 3 sensors measurement variations, estimated and model output using conventional Kalman algorithm. $\mathrm{O}_{2}$, BZT, KIT measurements deviates from zero during 200-300 and 400-500 sampling interval with the magnitude of $10 \%, 150^{\circ} \mathrm{C}, 120^{\circ} \mathrm{C}$ and its estimated signal also deviating from the nominal value due to the faulty measurements. The corresponding residual signal is shown in Figure 6 and it is greater than the threshold value, so it is concluded that fault occurs during that period. Since the estimated output deviates from its nominal value due to faults, it cannot be used as the feedback signal to the controller, so it is necessary to generate the replacement signal during that faulty period.

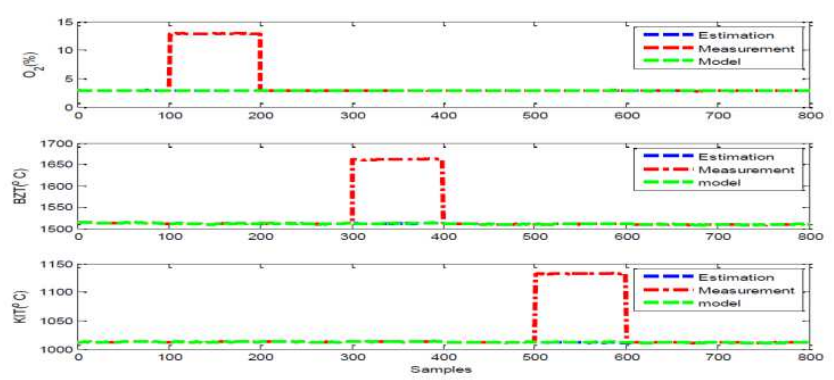

Figure 7: Sensor Measurements under Bias Fault
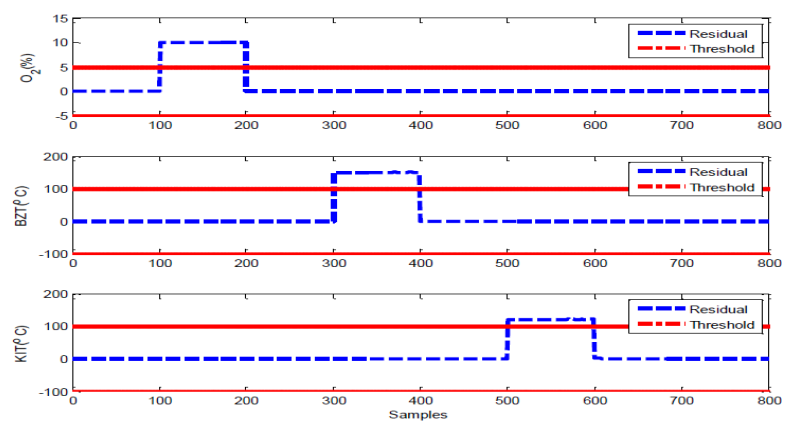

Figure 8: Residual Signals under Bias Fault

Using the proposed Kalman algorithm, the replacement signals have been generated and shown in Figure 7. It is observed that, estimated signal believes both measurement signal and model under the faultfree condition. During 100-200, 300-400, 500-600 sampling interval, sensor measurements suddenly increases from its nominal value. $\mathrm{O}_{2}$ signal increases with the magnitude of 10\% from its nominal value during 100-200 interval, during 300-400 sampling interval, BZT signal 
increase from its nominal value with the magnitude of $150^{\circ} \mathrm{C}$ and KIT signal increases from its nominal value with the magnitude of $120^{\circ} \mathrm{C}$ from its nominal value during 500-600 sampling interval. During the faulty period, estimated signal follows the model output, not the faulty measurements and this predicted output will be used as the replacement signal in order to give fault free feedback signal to the controller. The corresponding residual signal is shown in Figure 8, it is observed that this signal is above the thres hold value, so it is concluded that fault occurs.
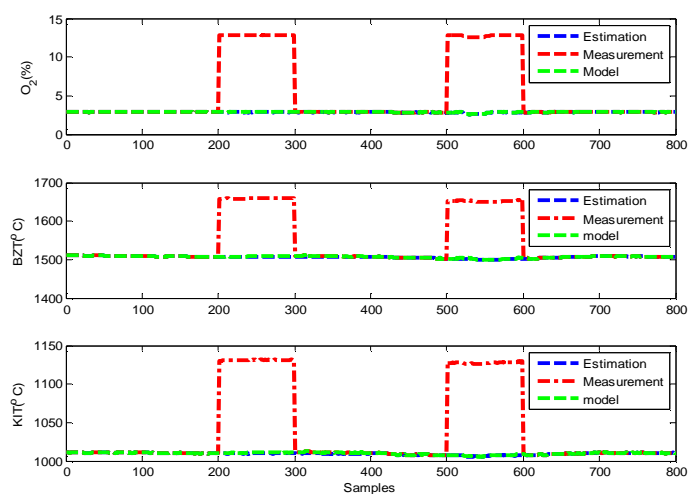

Figure 9: Sensor Measurements under Input Disturbance and Bias Fault

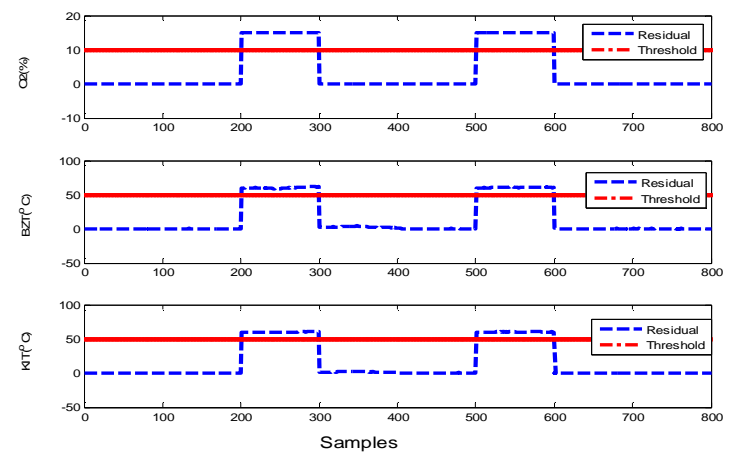

Figure 10: Residual Signals under Input Disturbance and Bias Fault

Figure 9 shows that estimated signal and model output slightly deviating from its nominal value and comes back to its original value during 520-560 sampling interval. The estimated signal is not following faulty sensor measurements and it follows the model output, which never changes with respect to fault, so it is concluded that the deviation is due to the disturbance at the input not due to fault

Figure 10 shows the residual signal under disturbance at the input and it is observed that the residual is not changing with respect to input disturbance. The residual signal is above the threshold and maintained at the constant value for a particular period, so bias fault concluded.

Figure 11 shows three sensors state estimation, measurement variations and model output.During 200-300 sampling interval, an $\mathrm{O}_{2}$ measurement signal is suddenly deviating from its nominal value to $10 \%$, so it is concluded that bias the fault occurs. At 520th sample, $\mathrm{O}_{2}$ measurement and its estimation, model output slightly deviating from its nominal value and comes back to its original, this deviation is due to the disturbance at the input not due to fault because model output never changes for fault occurrence.In BZT measurement no fault occurs because no sudden deviation of 
measurement from its nominal value, during 520-560 sampling interval, measurements, model, estimation slightly deviating from its nominal and comes back to its original and this deviation is due to the disturbance at input not due to fault because model output never change with fault at output sensor.In KIT measurement, bias fault occurs during 200-300 sampling interval with the amplitude of $120^{\circ} \mathrm{C}$ and the temperature completely decreases to zero during 500-600 sampling interval, so it is concluded that complete sensor measurement failure occurs.
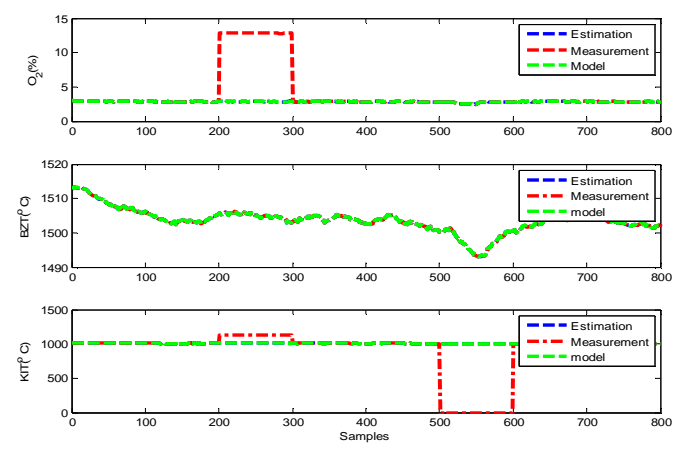

Figure 11: Sensor Measurements under Multiple Faults
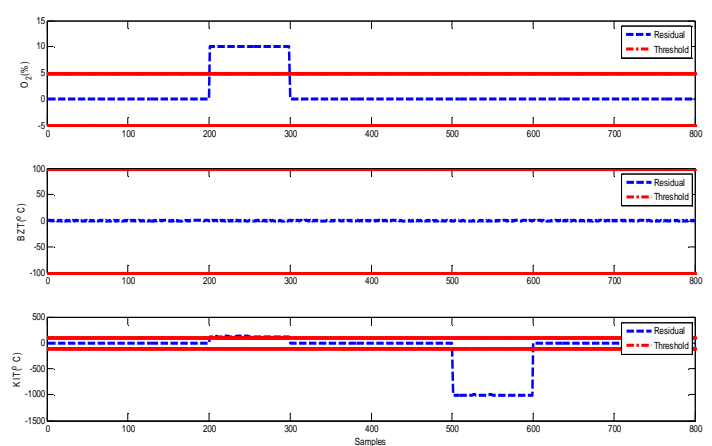

Figure 12: Residual Signals under Multiple Faults

\section{CONCLUSIONS}

In this work, Kalman filter based estimation technique has been used for detecting faults and generating replacement signal for pyrometer, thermocouple, and oxygen analyzer measurements in kiln process. Since the Kalman algorithm needs the model of the system, system identification tool in MATLAB was used for identifying subspace method based state space model of sensors. Bias fault and sensor measurement failure have been analyzed using Kalman based residual generation method by finding the difference between the estimated value from the actual measurements. From the results, it was observed that there is a deviation in the residual signal of KIT, BZT, $\mathrm{O}_{2}$ from zero and it indicates that the sensor is affected by the fault. This algorithm also generates replacement signal from the predicted output irrespective of the measurements during the faulty condition. So, from these results, it is concluded that the sensor measurement faults have been detected from the residual signal and replacement signal is generated by prediction not by correction during the faulty condition in order to avoid the degradation of controller performance. 


\section{REFERENCES}

1. Gilbert Hock Beng Foo, Xinan Zhang, and D MahindaVilathgamuwa, "A sensor fault detection and isolation method in interior permanent-magnet synchronous motor drives based on an Extended Kalmanfilter", IEEE Transactions on Industrial Electronics, 60(8):3485-3495, 2013.

2. V Gomathi, SeshadhriSrinivasan, $K$ Ramkumar, and GuruprasathMuralidharan, "Structural analysisbased sensor measurement fault diagnosis in cement industries”, Control Engineering Practice, 64:148-159, 2017.

3. ChingizHajiyev, "Generalized Rayleigh quotient based innovation covariance testing applied to sensor/actuator fault detection”, Measurement, 47:804-812, 2014.

4. A.Hsoumi, R.ElHarabi, S.B.H.Ali, and M.N. Abdelkrim, "Diagnosis of a continuous stirred tank reactor using Kalman filter", In Computational Intelligence, Modelling and Simulation, 2009,, International conference on CSSim'09, pages 153-158,Sept 2009.

5. Sunan Huang, KokKiong Tan, and Tong HengLee, "Fault diagnosis and fault-tolerant control in linear drives using the Kalmanfilter”, Industrial Electronics, IEEE Transactions on, 59(11):4285-4292, Nov 2012.

6. Rolf Isermann, "Fault-Diagnosis Systems: An Introduction from Fault Detection to Fault Tolerance, chapter Introduction”, pages 1-10. Springer Berlin Heidelberg, Berlin, Heidelberg, 2006.

7. Sanjay Jayaram, “A new fast converging Kalmanfilter for sensor fault detection and isolation”. Sensor Review, 30(3):219-224, 2010.

8. Jongchul Jung, Sangoh Han, and KunsooHuh, "Robust proportional-integral Kalmanfilter design using a convex optimization method”, Journal of Mechanical Science and Technology, 22(5):879-886, 2008.

9. T. Matias, D. Gabriel, F. Souza, R. Araujo, and J. Costa Pereira, "Fault detection and replacement of a temperature sensor in a cement rotary kiln”, In Emerging Technologies Factory Automation (ETFA), 2013 IEEE 18th Conference on, pages 1-8, Sept 2013.

10. A.T. Nair, T.K. Radhakrishnan, K. Srinivasan, and S. RominusValsalam, "Kalmanfilter based state estimation of a thermal power plant”,In Process Automation, Control and Computing (PACC), 2011 International Conference on, pages 1-5, July 2011.

11. O. Ondel, E. Boutleux, Eric Blanco, and G. Clerc, "Coupling pattern recognition with state estimation using Kalmanfilter for fault diagnosis", Industrial Electronics, IEEE Transactions on, 59(11):4293-4300, Nov 2012.

12. TeerawatSangpet, SuwatKuntanapreeda, and RudigerSchmidt, "Hysteretic nonlinearity observer design based on Kalmanfilter for piezo-actuated flexible beams with control applications", International Journal of Automation and Computing, 11(6):627634, 2015.

13. Mitch Serpas, Yunfei Chu, and Juergen Hahn, "Fault detection approach for systems involving soft sensors", Journal of Loss Prevention in the Process Industries, 26(3):443 - 452, 2013. Papers presented at the 2011 Mary Kay OConnor Process Safety Center International Symposium.

14. Bruno Sinopoli, Luca Schenato, Massimo Franceschetti, KameshwarPoolla, Michael I Jordan, and Shankar S Sastry, “Kalmanfiltering with intermittent observations", IEEE transactions on Automatic Control, 49(9):1453-1464, 2004.

15. HalilErsinSoken, ChingizHajiyev, and Shinichiro Sakai, "Robust Kalmanfiltering for small satellite attitude estimation in the presence of measurement faults", European Journal of Control, 20(2):64 - 72, 2014. 
16. VenkatVenkatasubramanian, RaghunathanRengaswamy, and Surya $N$ Kavuri, "A review of process fault detection and diagnosis: Part ii: Qualitative models and search strategies”, Computers \& Chemical Engineering, 27(3):313 - 326, 2003.

17. VenkatVenkatasubramanian, RaghunathanRengaswamy, Surya N. Kavuri, and Kewen Yin, "A review of process fault detection and diagnosis: Part iii: Process history based methods”, Computers \& Chemical Engineering, 27(3):327 - 346, 2003 .

18. VenkatVenkatasubramanian, RaghunathanRengaswamy, Kewen Yin, and Surya N. Kavuri, "A review of process fault detection and diagnosis: Part I Quantitative model-based methods", Computers \& Chemical Engineering, 27(3):293 - 311, 2003 .

19. Kris Villez, BabjiSrinivasan, RaghunathanRengaswamy, Shankar Narasimhan, and VenkatVenkatasubramanian. Kalmanbased strategies for fault detection and identification (fdi): Extensions and critical evaluation for a buffer tank system. Computers \& Chemical Engineering, 35(5):806 - 816, 2011. Selected Papers from ESCAPE-20 (European Symposium of Computer Aided Process Engineering - 20), 6-9 June 2010, Ischia, Italy.

20. XiulingXu and Xiaodong Wang. Intelligent Computing and Information Science: International Conference, ICICIS 2011, Chongqing, China, January 8-9, 2011. Proceedings, Part I, chapter Hard-Failure Diagnosis Using Self-tuning Kalman Filter, pages 326-334. Springer Berlin Heidelberg, Berlin, Heidelberg, 2011.

21. Yucheng Zhou, JiaheXu, and Yuanwei Jing. Comparison of centralized multi-sensor measurement and state fusion methods with ensemble Kalmanfilter for process fault diagnosis. In Control and Decision Conference (CCDC), 2010 Chinese, pages 3302-3307, May 2010. 